Kristallisation aus Lösungen (G. T. Kohman), Wachstum aus der Schmelze (W. A. Tiller), und Grundlagen der Rekristallisation (W. G. Burgers).

Die generelle Einleitung zur Lösungskristallisation wird durch einen höchst aktuellen und bemerkenswerten Beitrag über Dampfphasen-Reaktionen zwischen Metall. halogeniden und Wasser ergänzt (R. O. Grisdale).

An die Hauptberichte werden jeweils Referate über Wachstum und Züchtung spezieller Kristallarten angeschlossen. Im einzelnen werden behandelt: Metalle (S. S. Brenner), organische Verbindungen (R. S. Bradley), Sulfide (D. C. Reynolds), Silicium (R. Glang, E. S. Wajda), SiC (J. R. O'Connor), Fis (B. J. Mason) bei der Dampfphasenkristallisation, Elemente (R. H. Wentorf, Jr.), Ionenkristalle (P. H. Egli, L. R. Johnson), Silberhalogenide (C. Berry, W. West, F. Moser), hydrothermales Wachstum (A. A. Ballman, R. A. Laudise), Schmelzflusslösungen (R. A. Laudise) bei der Lösungskristallisation, Elemente mit niedrigen Schmelzpunkt (A. J. Goss), hochschmelzende Elemente (H. W. Schadler), Halbleiter (W. D. Lawson, S. Nielsen) III-V-Verbindungen (L. Weisberg), Verneuil-Methode (W. H. Bauer, W. G. Field), Alkalihalogenide (R. W. Dreyfus) bei der Solidifikation und schliesslich Wachstum grosser Kristalle durch Rekristallisation (K. T. Aust).

In diesen Beiträgen hervorragender Experten sind zahlreiche Details mit bemerkenswerten Akzenten enthalten. Es sei das epitaktische Wachstum von Metallfilmen, von Halbleiterschichten und von Eis auf verschiedenen Substraten erwähnt. Die Herstellung von Yttrium-Eisen-Granat durch Hydrothermalsynthese oder aus Schmelzflusslösungen und die Entwicklung neuerer Heizungsquellen bei der Verneuil-Methode (Flammenbogen-Abbildung, Plasma u. a.) sind nur einige wenige Beispiele, die ein lebhaftes Interesse erregen werden.

W. KLEBER

Institut für Mineralogie

Humboldt-Universität zu Berlin

Invalidenstrasse 43

Berlin, N4

Deutschland

\section{The effects of ultrasound on the kinetics of} crystallization. By A. P. KaPustin. $65 \mathrm{pp}$. New York: Consultants Bureau, 1963. Price $\$ 12 \cdot 50$.

Die hohe wissenschaftlich-technische Bedeutung in der Anwendung des Ultraschalls ist heute unbestritten. Der hauptsächliche Effekt des Ultraschalls auf Materie beruht in der Erzeugung von Druckwellen mit beachtlichen Amplituden von einigen atm. Eine besondere Rolle spielt dabei die Kavitation durch intensive Ultraschall-Impulse. Beim Kollaps der Kavitäten können sekundäre Schockwellen entstehen, deren Drucke jene der Schallwellen um ein Vielfaches übersteigen können. Es ist verständlich, dass die Anwendung des Ultraschalls - in den letzten 15 Jahren etwa - auch auf kristallographischem Gebiet in zunehmendem Masse Eingang gefunden hat. Objekte der Ultraschall-Untersuchungen sind Einkristalle und Kristall-Aggregate, sowie die Prozesse der Kristallisation, der Keimbildung, des Kristallwachstums und der Auflösung bzw. Ätzung von Kristallen.

Die vorliegende Abhandlung berichtet im wesentlichen über die Arbeiten des Verfassers und seiner Mitarbeiter am Institut für Kristallographie der Akademie der Wissenschaften der UdSSR in Moskau. Sie betreffen die experimentelle Seite der Kristallisation und der Dissolution von Kristallen in Ultraschall-Feldern verschiedener Frequenzen und Intensitäten. Auch einige Effekte, die in kristallinen Materialien erzeugt wurden, werden beschrieben.

Nach einem Überblick über Methoden und Apparaturen für das Studium des Wachstums und der Auflösung von Kristallen in Ultraschall-Feldern folgt eine kurzer Bericht über Arbeiten, die sich mit dem gestellten Thema beschäftigen. Ein ausführliches Kapitel wird dann Kristallisations-Prozessen organischer Verbindungen gewidmet. Es braucht kaum betont zu werden, dass die Keimbildung unter dem Einfluss eines Ultraschall-Feldes ein besonders interessantes Ciebiet darstellt.

Im einzelnen wird über die Wirkung von Ultraschall. wellen auf das Wachstum von Kalialaun und von Metall-Einkristallen berichtet. Schliesslich werden Fragen der Auflösung, der Bildung von Ätzfiguren, des Nachweises von Versetzungen und des Verhaltens kristalliner Flüssigkeiten in Anwesenheit von Ultraschall diskutiert.

Das Problem der Einwirkung von Ultraschall-Feldern auf die Vorgänge des Wachstums, der Dissolution und der Transformationen von Kristallen ist höchst komplexer Natur und deshalb heute theoretisch kaum auch nur in Ansätzen zu lösen. Die bedeutsamen technischen Aspekte fordern nachdrücklich eine Intensivierung der experimentellen Forschung auf dem behandelten Gebiet, und es ist zu wünschen, dass die vorgelegte Monographie Untersuchungen im Sinne des Autors stimuliert.

W. KLEBER

Institut für Mineralogie

Humboldt-Universität zu Berlin

Invalidenstrasse 43

Berlin, N4

Deutschland

The physics and chemistry of ceramics. Proceedings of a symposium held at The Pennsylvania State University May 28-30. 1962. Edited by C. Klingsberg. Pp. 361. New York and London: Gordon \& Breach, Science Publishers, 1963. Price $\$ 14.50$ (cloth), $\$ 9.50$ (paper).

Aus zwei Gründen wurde dieses Symposium veranstaltet. Einmal sollten Festkörper-Physiker und -Chemiker mit Keramikern zusammengeführt werden, um das Gemeinsame in den Forschungsrichtungen festzustellen, was zu einer engeren Zusammenarbeit Anlass geben soll. Zum anderen war in diesem Kreis eine Diskussion über die Frage vorgesehen, ob in den U.S.A. die gegenwärtige Hochschulausbildung in der Fachrichtung Keramik den Anforderungen der Zukunft gewachsen ist. Entsprechend gliedert sich der Inhalt dieses Buches in zwei Teile, wobei die im Wortlaut wiedergegebenen wissenschaftlichen Vorträge und Diskussionen des ersten Teils den grössten Raum einnehmen. Sie behandeln folgende Themen :

F. Seitz: The interrelation of ceramics, metallurgy, chemistry and physics. 